\title{
Observer preference for a dedicated medical display vs a standard screen in the detection of dental radioanatomic features
}

\author{
Sohaib Shujaat, MSC (Dent. Sci.), OMFS, ${ }^{\mathrm{a}}$ Carolina Letelier, DDS, ${ }^{\mathrm{a}, \mathrm{b}}$ Annelore De Grauwe, MSC, \\ Karla de Faria Vasconcelos, Phd, ${ }^{\text {a,c }}$ Berkan Celikten, Phd, ${ }^{a}$ and Reinhilde Jacobs, Phd ${ }^{\mathrm{a}, \mathrm{d}}$
}

Objective. The aim of this study was to assess observers' preference for a dentomaxillofacial dedicated medical display (MD) vs a general-purpose standard screen (SS) for in vitro and in vivo observation of normal radioanatomic features.

Study Design. The in vitro sample consisted of 2-dimensional (2-D) intraoral $(n=15)$, panoramic $(n=2)$, cephalometric $(n=2)$, and 3-dimensional (3-D) cone beam computed tomography (CBCT) $(n=9)$ data sets, acquired by utilizing commercially available skull and head-and-neck phantoms. The in vivo sample consisted of 80 radiographs (intraoral =20; panoramic $=20$; cephalometric $=20$; and $\mathrm{CBCT}=20$ ). In vitro and in vivo data sets were both acquired by using Minray, Promax2-D, and Vistapano Ceph for 2-D images and Accuitomo, NewTom VGi evo, and Promax3-D for CBCT images. Five observers entered screen preferences when evaluating the appearance of radioanatomic structures on MD and SS.

Results. Both in vitro and in vivo assessments showed good interobserver and excellent intraobserver agreement. In vitro data suggested a significant preference for MD over SS for viewing radioanatomic features on panoramic and CBCT images, whereas MD was significantly preferred for in vivo images of all imaging modalities $(P<.001)$.

Conclusions. Overall, observers preferred MD over SS for both in vitro and in vivo observation of normal radioanatomic features irrespective of the imaging modality. (Oral Surg Oral Med Oral Pathol Oral Radiol 2020;000:1-8)

Radiography is an integral component in the diagnosis and treatment planning of dentomaxillofacial pathoses. ${ }^{1}$ Recent advances in digital dental imaging have led to a paradigm-changing revolution in dentistry. Digital intraoral (IO) and panoramic (Pan) radiography has replaced film-based radiography as the basic imaging modality for 2-dimensional (2-D) evaluation of radioanatomic structures, whereas cone beam computed tomography (CBCT) imaging has been accepted as a primary diagnostic tool for 3-dimensional (3-D) evaluation. ${ }^{2}$ The adoption of digital radiography has eliminated the chemical developing process and reduced environmental contamination significantly. ${ }^{3,4}$

In addition to image postprocessing techniques and enhancement filters, ${ }^{5-7}$ an important aspect for optimization of the digital dental workflow is related to computer display screens. According to American College of Radiology and the U.S. Food and Drug Administration, display screens can be classified as either primary or secondary screens. Primary screens are used by

aOMFS-IMPATH Research Group, Department of Imaging \& Pathology, Faculty of Medicine, KU Leuven \& Oral and Maxillofacial Surgery, University Hospitals Leuven, Leuven, Belgium.

${ }^{\mathrm{b}}$ Department of Oral and Maxillofacial Radiology, University of Los Andes, Santiago, Chile.

${ }^{\mathrm{c}}$ Department of Oral Diagnosis, Division of Oral Radiology, Piracicaba Dental School, State University of Campinas, Sao Paulo, Brazil. ${ }^{\mathrm{d}}$ Division of Oral Diagnostics and Rehabilitation, Department of Dental Medicine, Karolinska Institutet, Sweden.

Received for publication Oct 3, 2019; returned for revision Feb 7 , 2020; accepted for publication Feb 14, 2020.

(c) 2020 Elsevier Inc. All rights reserved.

2212-4403/\$-see front matter

https://doi.org/10.1016/j.00oo.2020.02.011 radiologists for interpreting medical images, whereas secondary screens are utilized by clinicians for viewing images after interpretation by a radiologist on a primary screen. ${ }^{8}$ Image display is a vital step in the digital imaging chain, and any flaw in the display can directly affect the quality and diagnostic efficacy of an image.

In medical and dental imaging, accurate radiographic representation of anatomic structures is of great importance. Over the past few years, liquid crystal display (LCD) screens have almost completely replaced cathode-ray tubes (CRT) in radiology. ${ }^{8}$ The key display characteristics of screens, such as noise, resolution, luminance response and uniformity, pixel size, geometric distortion, and display reflection, impact image quality, thereby affecting observer performance. ${ }^{8,10}$ The utilization of dedicated medical display (MD) screens that optimize these characteristics has been well documented and has shown improved diagnostic and clinical performance in observing breast cancer on screening mammograms and lung nodules on chest radiographs. ${ }^{11,12}$

MD screens are available in dentomaxillofacial imaging. Dentally configured MDs have been engineered on the basis of the resolution and gray scale of

\section{Statement of Clinical Relevance}

The quality of image displays in dentomaxillofacial radiology may affect the efficacy of radiographic interpretation, diagnosis, and treatment planning. Dedicated medical display devices may improve diagnostic efficacy compared with standard screens. 
dental radiographs, unlike normal MDs that are configured on the basis of medical radiology images. ${ }^{13-15}$ However, it is questionable whether specifically configured "dental" settings on an MD are actually beneficial in such tasks as detection of radioanatomic structures and diagnosis of disease. There is lack of scientific evidence to support the notion that use of these monitors improves efficacy in observing radioanatomic features compared with general purpose standard screens (SS).

Therefore, the aim of the present study was to assess observers' preference for a dentomaxillofacial dedicated MD vs a general-purpose SS for in vitro and in vivo observation of normal radioanatomic features on 2-D and 3-D images.

\section{MATERIALS AND METHODS \\ Ethical approval}

This retrospective study was conducted in compliance with the tenets of the World Medical Association Declaration of Helsinki on medical research. Ethical approval was acquired from the Medical Ethics Committee of University Hospitals, KU Leuven, Leuven, Belgium (Reference No. S59247). Patient-specific images and information were anonymized. In vitro and in vivo samples of radiographs were collected for this study. An in vitro sample was evaluated because it allowed image analysis without patient motion artifacts, whereas the in vivo images represented actual clinical conditions. Inclusion criteria were good quality of images and the presence of sharply delineated normal radioanatomic features. Exclusion criteria were presence of any metal, restoration, and/or motion artefacts affecting image quality

\section{In vitro sample}

A commercially available skull phantom, covered with Mix D soft tissue simulation, ${ }^{16}$ was placed in a Minray IO radiographic unit (Soredex, Tuusala, Finland) operating at $65 \mathrm{kVp}$ and $7 \mathrm{~mA}$ for acquiring IO radiographs with use of the paralleling technique $(\mathrm{n}=15)$. A full head-and-neck Rando phantom (Alderson-Rando, Long Beach, CA) with soft tissue simulation was placed in 2 radiographic devices, the Promax 2-D (Planmeca, Helsinki, Finland) and the Vistapano Ceph (Durr Dental, Bittigheim-Bissingen, Germany) to generate one Pan and one cephalometric (Ceph) radiograph with each device $(n=2$ Pan and $n=2$ Ceph). Three CBCT devices, 3-D Accuitomo 170 (J. Morita, Kyoto, Japan), NewTom VGi evo (QR, Verona, Italy), and Promax 3-D Max (Planmeca, Helsinki, Finland), with 9 overall protocols consisting of combinations of fields of view (FOVs), exposure doses, and voxel sizes, were utilized for generating 3-D images of the phantom head and neck.

\section{In vivo sample}

A retrospective sample of radiographs from 80 patients referred to the dentomaxillofacial radiology center (University Hospitals Leuven, Belgium) for 2-D and 3D diagnostic imaging was included for the in vivo evaluation. Radioanatomic features were observed on IO $(n=20)$, Pan $(n=20)$, Ceph $(n=20)$ radiographs and on the axial, sagittal, and coronal views of 20 randomly selected CBCT scans acquired by using the same acquisition devices.

For both in vitro and in vivo images, detection of radioanatomic features on the IO, Pan, and CBCT radiographs included the cementoenamel junction (CEJ); pulpal canal (PC) structure; apical third of the pulp canal (APC; defined as the apical one-third of the canal between the CEJ and root apex); periodontal ligament space; lamina dura; alveolar crest (AC); and trabecular structure. On the Ceph radiographs, point $\mathrm{A}$, point B, and soft tissue were observed (Table I). Radioanatomic features were observed around each tooth on all 2-D radiographs. The 9 in vitro CBCT image data sets, corresponding to the 9 protocols, were evaluated on the axial, sagittal, and coronal planes around 4 maxillary teeth, that is, left and right central incisors and canines ( 9 protocols $\times 4$ teeth $=36$ images); and 3 mandibular teeth, that is, the left first premolar, right second premolar, and right first molar ( 9 protocols $\times 3$ teeth $=27$ images). The CBCT units and the 9 protocols used are presented in Table II.

\section{Display screens}

The first screen was a dedicated MD Barco MDRC2221; (Barco, Kortrijk, Belgium), calibrated in the DICOM (Digital Imaging and Communications in Medicine) format with interior compensation light and frontal sensor that overcame inherent instability of backlight lamps. The second screen was a general-purpose SS Dell U2415 b UltraSharp Widescreen LCD (Dell Inc., Round Rock, TX). Table III describes the

Table I. Normal radioanatomic structures

\begin{tabular}{ll}
\hline Type of modality & Anatomic features \\
\hline Intraoral Panoramic & Cementoenamel junction \\
Cone beam computed & (CEJ) \\
tomography & Pulpal canal (PC) structure \\
& Apical third of pulp canal \\
& (APC) \\
& Periodontal ligament space \\
& (PLS) \\
& Lamina dura (LD) \\
& Alveolar crest (AC) \\
& Trabecular structure (TS) \\
Cephalometric & Point A \\
& Point B \\
& Soft tissue \\
\hline
\end{tabular}


Table II. CBCT acquisition devices and protocols

\begin{tabular}{lll}
\hline CBCT & FOV & Voxel Size $(\mu \mathrm{m})$ \\
\hline 3-D Accuitomo 170 & $4 \times 4 \mathrm{~cm}$ & 80 \\
(J. Morita, Kyoto, & $6 \times 6 \mathrm{~cm}$ & 125 \\
Japan) & $8 \times 8 \mathrm{~cm}$ & 160 \\
NewTom VGi evo & $8 \times 5 \mathrm{~cm} \mathrm{RS}$ & 200 \\
(QR, Verona, Italy) & $8 \times 8 \mathrm{~cm} \mathrm{RS}$ & 200 \\
& $8 \times 8 \mathrm{~cm} \mathrm{RECO}$ & 200 \\
Promax 3-D Max & $10 \times 9 \mathrm{~cm}$ ULD LD & 400 \\
(Planmeca, Helsinki, & $10 \times 9 \mathrm{~cm}$ ULD HD & 150 \\
Finland) & $10 \times 9 \mathrm{~cm}$ NHD & 150
\end{tabular}

$C B C T$, cone beam computed tomography; FOV, field of view; $N H D$, normal high definition; $R E C O$, regular economic quality; $R S$, regular standard quality; $U L D L D$, ultra-low dose low definition; $U L D H D$, ultra-low dose high definition.

specifications of the screens. The specified MD and SS were selected because these were the most commonly utilized primary screens in our radiology clinics for performing radiologic tasks. The main visual differences between the screens were related to size and contrast. However, the observers were unaware which screen was an MD or an SS based on size. The viewing angles for both screens were the same. Luminance for both screens was set at maximum to achieve optimal contrast ratio setting. Images were analyzed in a dimmed room at a $60 \mathrm{~cm}$ viewing distance.

\section{Observations}

Five independent blinded observers evaluated the images after initial training and calibration. The 5 observers were all specialists in oral and maxillofacial radiology and had a minimum of 5 years of experience in the field. A duplicated setup of the screens was configured to evaluate the same structures on both screens at the same time. The screen's contours and brand logos were hidden within a wall-mounted frame to blind the observers during image evaluation, and both screens were assigned separate numbers randomly for each observer. The images were preopened before being observed to further blind the observers to the type of screen.

Table III. Specifications of screens

\begin{tabular}{lll}
\hline Parameters & Medical display & Standard screen \\
\hline Display size, diagonal & $54.1 \mathrm{~cm}(21.3$ inch) & $61.1 \mathrm{~cm}(24.1 \mathrm{inch})$ \\
Technology & Thin-film transistor & In-plane switching \\
Resolution & $1600 \times 1200$ & $1920 \times 1200$ \\
Pixels per inch & 94 & 132 \\
Pixel pitch, mm & $0.27 \mathrm{~mm}$ & $0.27 \mathrm{~mm}$ \\
$\begin{array}{l}\text { Maximum brightness, } \\
\text { candela } / \mathrm{m}^{2}\end{array}$ & $440 \mathrm{~cd} / \mathrm{m}^{2} ;$ typical & $300 \mathrm{~cd} / \mathrm{m}^{2}$ (typical) \\
$\begin{array}{l}\text { Default contrast ratio } \\
\text { Response time }\end{array}$ & $1500: 1$ typical & $1000: 1$ (typical) \\
\hline
\end{tabular}

\section{Subjective visualization scale}

The observers were asked to evaluate each of the radioanatomic features on each imaging modality on each screen and indicate which screen was preferred for each feature. A 3-point ordinal scale $(1-3)$ was used to evaluate the preference of the observers with $1=\mathrm{MD}$ preferred; $2=\mathrm{MD}$ and SS equally preferred; and $3=\mathrm{SS}$ preferred.

All observers performed the assessment twice separately with a 1-week interval to allow for calculation of interobserver and intraobserver agreements. The assigned screen numbers and their placements were also randomized after 1 week.

\section{Statistical analysis}

Data were analyzed by using SPSS Statistics for Windows, version 22.0 (IBM Corp., Armonk, NY). The interobserver and intraobserver agreements of paired observers were calculated for each type of radiograph in the in vitro and in vivo data sets by using the kappa test, which was classified as follows: poor $(<0.20)$, fair $(0.21-0.40)$, moderate $(0.41-0.60)$, good $(0.61-0.80)$, and excellent $(0.81-1.00) .{ }^{17}$ All observer preferences were expressed as percentages by pooling the responses of observers for the detection of each anatomic feature related to both in vitro $(\mathrm{IO}=15$; $\mathrm{Pan}=2 ; \mathrm{Ceph}=2 ; \mathrm{CBCT}=9)$ and in vivo $(\mathrm{IO}=20$; $\mathrm{Pan}=20 ; \mathrm{Ceph}=20 ; \mathrm{CBCT}=20$ ) data sets. The significance for the preference of MD in radioanatomic feature detection was calculated by using Pearson's $\chi^{2}$ test. A value of $P<.001$ was considered statistically significant.

\section{RESULTS}

Both in vitro and in vivo assessments showed overall good interobserver agreement $(\kappa \geq 0.80)$ and excellent intraobserver agreement $(\kappa \geq 0.93)$ without significant differences among the observers.

\section{In vitro assessment}

Figure 1 illustrates the percentages of observers' preference for detecting radioanatomic features on IO radiographs, with greater preference for MD compared with SS for 5 of the 7 radioanatomic features. PC and APC exhibited equal preference for MD and SS in all evaluations. SS was not preferred over MD for any feature. On Pan radiographs, the AC scored 2 on the ordinal scale (MD and SS equally preferred) for $100 \%$ observations. CEJ and PC showed no more than $14 \%$ observer preference in favor of MD (Figure 2). Similar to the data for IO, however, SS was not preferred over MD for any of the landmarks. Point A was the only anatomic landmark on Ceph radiographs that showed a slight observer preference for $\mathrm{MD}$, whereas point $\mathrm{B}$ 




Fig. 1. In vitro radioanatomic feature detection on intraoral radiographs. $A C$, alveolar crest; $A P C$, apical pulp canal; $C E J$, cementoenamel junction; $L D$, lamina dura; $M D$, medical display; $P C$, pulp canal; $P L S$, periodontal ligament space; $S S$, standard screen; $T S$, trabecular structure.

and soft tissue were equally preferred by all observers; no observers preferred SS over MD (Figure 3).

Figure 4 illustrates the results of the evaluation of the CBCT scans overall for the 7 radioanatomic features. NewTom VGi evo (QR, Verona, Italy), Regular Standard (FOV: $8 \times 5 \mathrm{~cm}$; voxel size: $200 \mu \mathrm{m}$ ) showed the lowest observer preference $(8 \%)$ in favor of MD, whereas Promax 3-D Max (Planmeca, Helsinki, Finland) ultra-low-dose low definition (FOV: $10 \times 9 \mathrm{~cm}$; voxel size: $400 \mu \mathrm{m}$ ) showed highest preference at $42 \%$ (see Figure 4). Overall, the Newtom VGI evo CBCT device $(\mathrm{QR})$ received the lowest preference for $\mathrm{MD}$ regardless of FOV and voxel size compared with the other 2 CBCT scanners. No observations in favor of SS were detected.

The combined observations showed no significant preference of viewing anatomic features on MD vs SS for IO $(P=.064)$ and Ceph radiographs $(P=.387)$, but observers significantly preferred MD over SS for viewing features on Pan radiographs and CBCT images $(P \leq .001)$, as shown in Table IV.

\section{In vivo assessment}

Figures 5 and 6 illustrate the preferences of observers for detecting radioanatomic features on IO and Pan radiographs, respectively. Preference for MD was

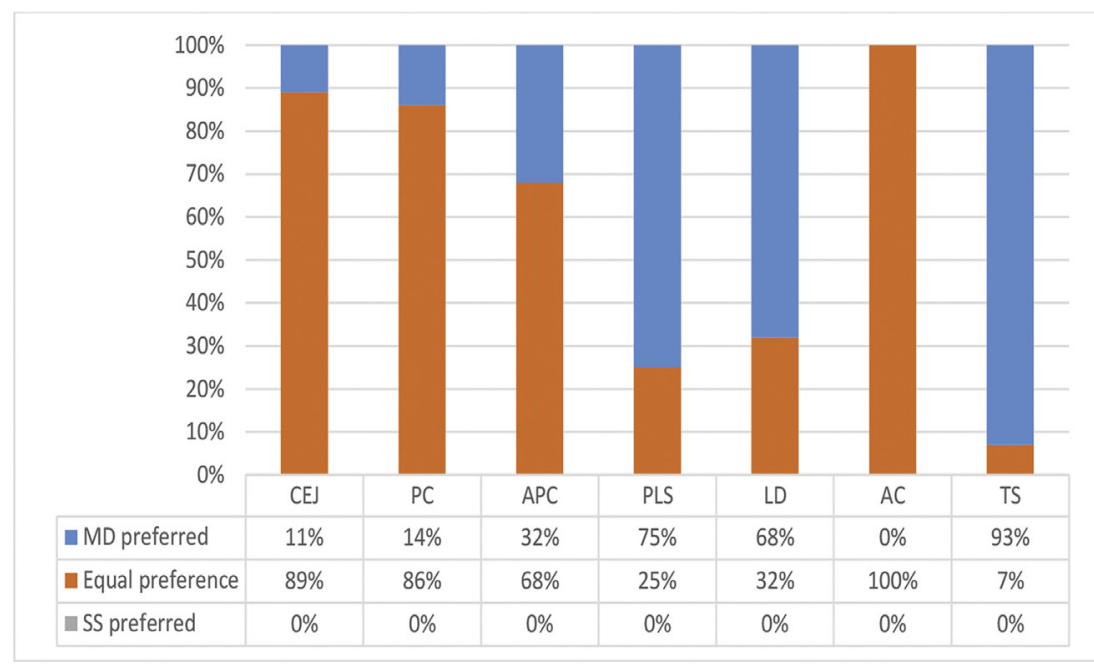

Fig. 2. In vitro radioanatomic feature detection on panoramic radiographs. $A C$, alveolar crest; $A P C$, apical pulp canal; $C E J$, cementoenamel junction; $L D$, lamina dura; $M D$, medical display; $P C$, pulp canal; $P L S$, periodontal ligament space; $S S$, standard screen; $T S$, trabecular structure. 


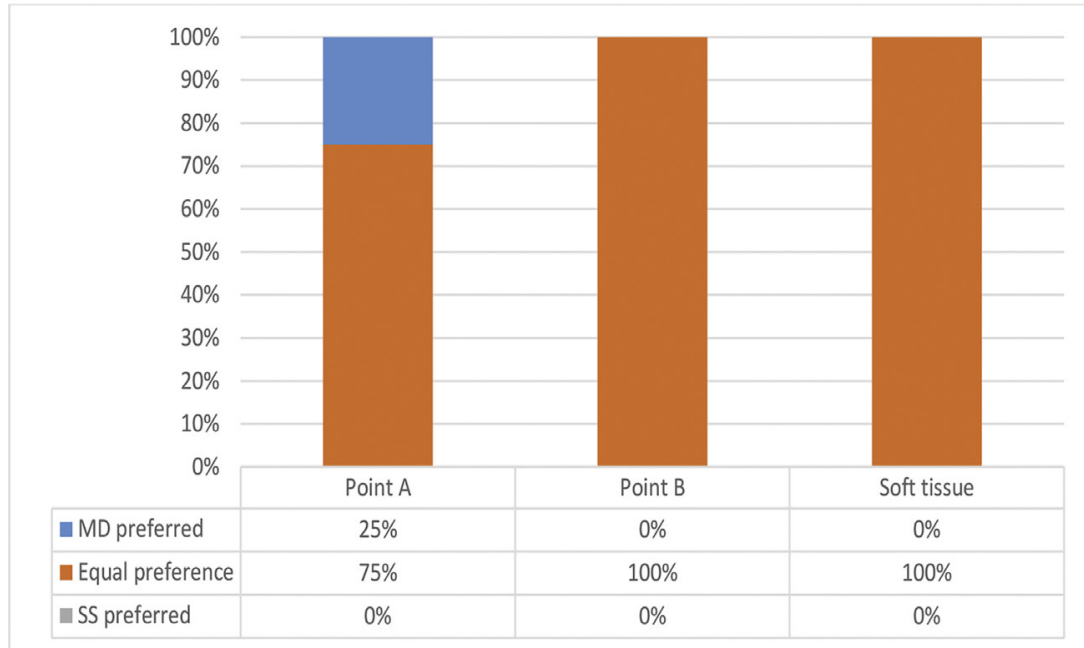

Fig. 3. In vitro radioanatomic feature detection on cephalometric radiographs. $M D$, medical display; $S S$, standard screen.

much greater than that for SS for all features in the IO series, with no observers preferring SS. Similar results were noted for the radioanatomic features on Pan radiographs, with the exception of the AC, for which only $5 \%$ expressed a preference for MD over SS. On Ceph radiographs, the observers preferred SS only in $2 \%$ of the data for detecting point A (Figure 7). The observers did not show a preference for SS for any of the CBCT images (Figure 8). Overall, a significant observer preference $(P \leq .001)$ was seen in favor of MD for detection of anatomic features with all imaging modalities (see Table IV).

\section{DISCUSSION}

The virtual imaging chain consists of 4 vital phases: raw data acquisition, data processing, electronic display, and visual interpretation by a viewer. With recent advancements in digital imaging, the influence of a display screen for increasing diagnostic efficacy, as evaluated through observer preference in detection of radioanatomic features, is of utmost importance. ${ }^{9}$ In the present study, in vitro data showed significant observer preference in favor of MD over SS when viewing anatomic structures on Pan radiographs and CBCT images. Analysis of in vivo radiographs revealed a significantly greater preference for MD over SS for all imaging modalities. In a large number of cases, both MD and SS were equally preferred, and in the rest, MD was given preference over SS by the observers. For the in vivo detection of the CEJ on IO radiographs, the observers showed the highest preference for detection with MD (88\%). Even though the

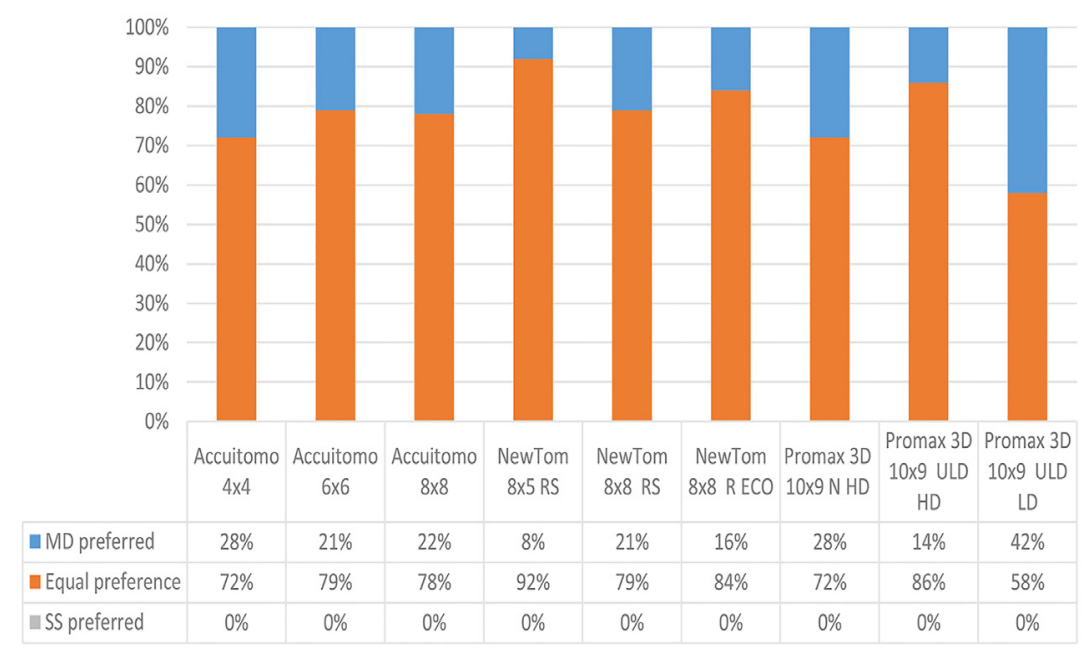

Fig. 4. In vitro radioanatomic feature detection on CBCT images with different CBCT devices and protocols. $M D$, medical display; $N H D$, normal high definition; $R E C O$, regular economic quality; $R S$, regular standard quality; $S S$, standard screen; $U L D H D$, ultra-low dose high definition; $U L D L D$, ultra-low dose low definition. 
Table IV. Overall screen preference for radioanatomic feature detection with all imaging modalities

\begin{tabular}{|c|c|c|c|c|c|c|c|c|}
\hline \multirow[t]{2}{*}{ Radiograph } & \multicolumn{3}{|c|}{ In vitro screen preference } & \multirow[t]{2}{*}{ P value } & \multicolumn{3}{|c|}{ In vivo screen preference } & \multirow[t]{2}{*}{$P$ value } \\
\hline & $M D$ & $E Q$ & SS & & $M D$ & $E Q$ & SS & \\
\hline Intraoral & $15 \%$ & $85 \%$ & $0 \%$ & .064 & $62 \%$ & $38 \%$ & $0 \%$ & $<.001$ \\
\hline Panoramic & $42 \%$ & $58 \%$ & $0 \%$ & $<.001$ & $43 \%$ & $57 \%$ & $0 \%$ & $<.001$ \\
\hline Cephalometric & $8 \%$ & $92 \%$ & $0 \%$ & .387 & $32 \%$ & $67 \%$ & $1 \%$ & $<.001$ \\
\hline CBCT & $22 \%$ & $78 \%$ & $0 \%$ & $<.001$ & $54 \%$ & $46 \%$ & $0 \%$ & $<.001$ \\
\hline
\end{tabular}

$C B C T$, cone beam computed tomography; $E Q$, equal preference; $M D$, medical display; $S S$, standard screen.

$\boldsymbol{P}$ value $<. \mathbf{0 0 1}=$ Significant preference in favor of medical display for detection of radioanatomic features.

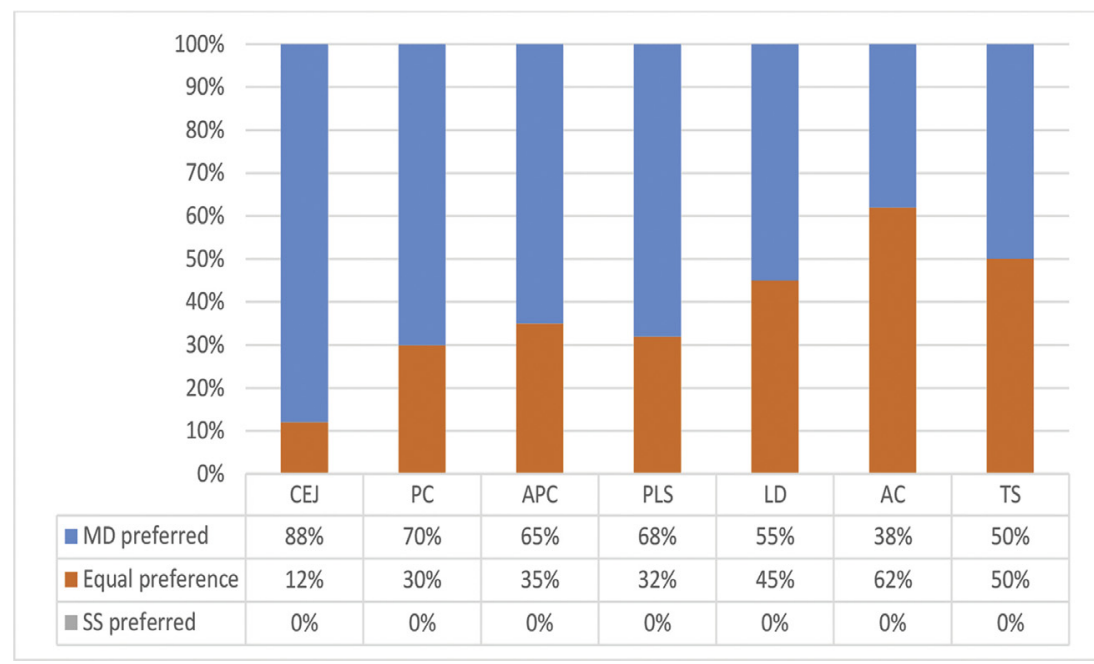

Fig. 5. In vivo radioanatomic feature detection on intraoral radiographs. $A C$, alveolar crest; $A P C$, apical pulp canal; $C E J$, cementoenamel junction; $L D$, lamina dura; $M D$, medical display; $P C$, pulp canal; $P L S$, periodontal ligament space; $S S$, standard screen; $T S$, trabecular structure.

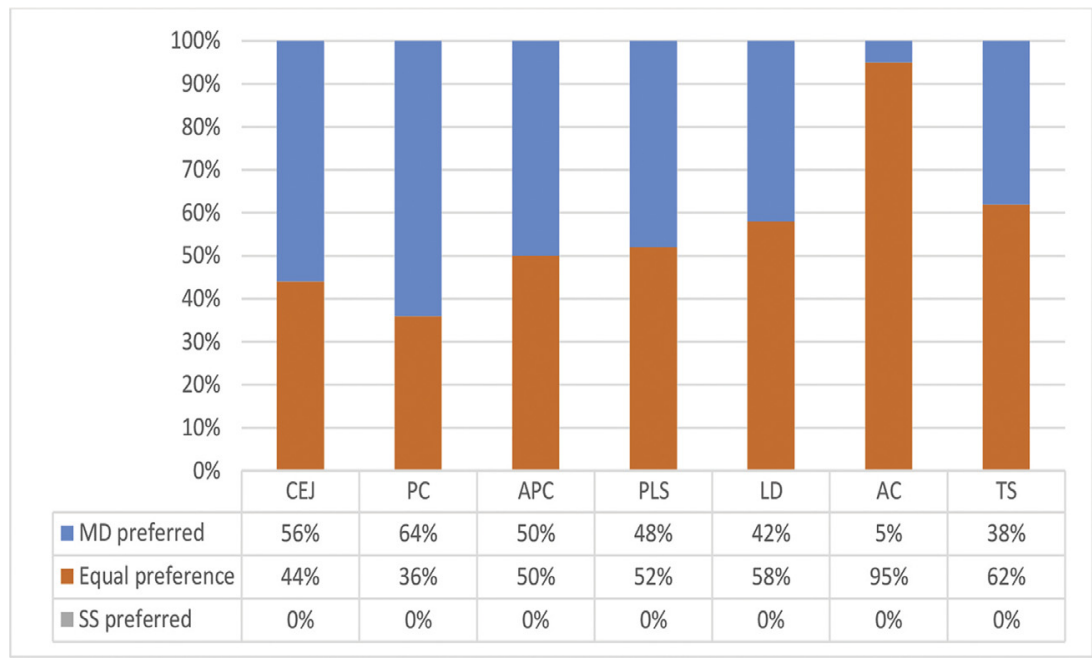

Fig. 6. In vivo radioanatomic feature detection on panoramic radiographs. $A C$, alveolar crest; $A P C$, apical pulp canal; $C E J$, cementoenamel junction; $L D$, lamina dura; $M D$, medical display; $P C$, pulp canal; $P L S$, periodontal ligament space; $S S$, standard screen; $T S$, trabecular structure. 


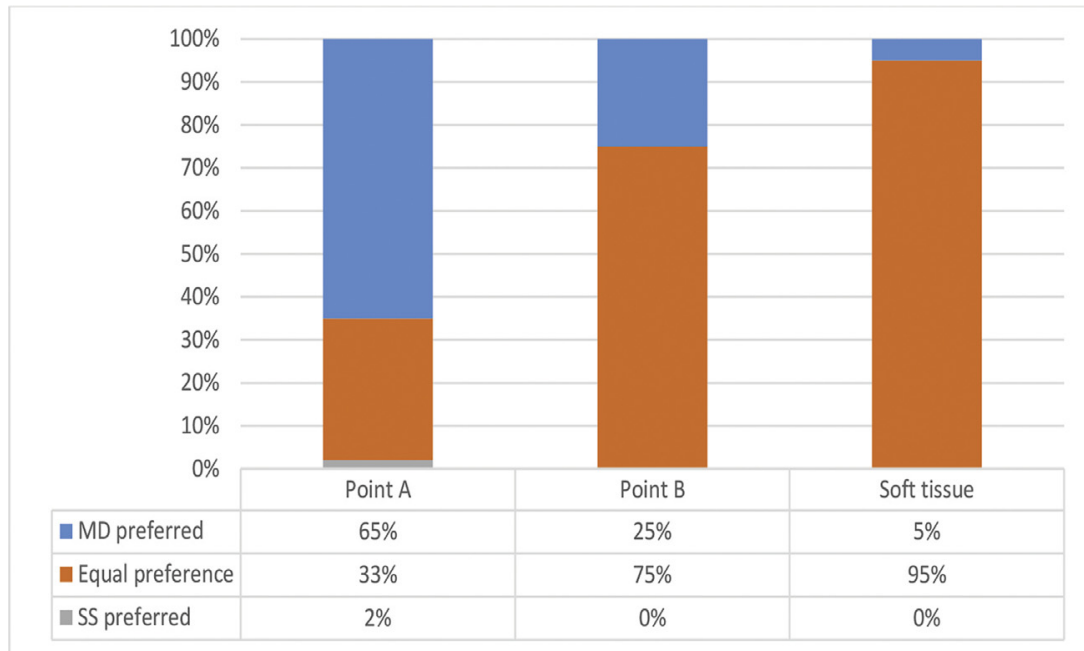

Fig. 7. In vivo radioanatomic feature detection on cephalometric radiographs. $M D$, medical display; $S S$, standard screen.

observers preferred both screens equally for the identification of the AC region on Pan radiographs (95\%), the performance of the observers when using SS might be reduced as teeth often hide the crestal area because of normal distortion related to the technique. ${ }^{18}$ According to Kallio-Pulkkinen et al., detection of anatomic structures on Pan radiographs relied on the experience of the observers, with the more experienced observers detecting radioanatomic features on both consumergrade and dedicated MDs without significant difference. ${ }^{19}$ In contrast, all of the experienced observers in our study preferred detection of radioanatomic feature on MD over SS with preinstalled dental settings.

The analysis of Ceph images mainly showed a difference in preference for detecting point A. However, observers considered MD as the preferred screen in assessing both in vitro and in vivo images. Because point $\mathrm{A}$ is the most difficult landmark to detect, ${ }^{20} \mathrm{MD}$ can be useful in this situation, especially for assessing landmarks on Ceph radiographs with low resolution. On the basis of our findings, high feature detection became more dependent on the CBCT device and its capacity to acquire, process, and show images on the screen. In contrast, CBCT images with low-dose protocols, lower resolution, and greater voxel size provided improved detection of radioanatomic features by the observers on MD. This might be related to the improved luminance stabilization and the special DICOM format of MD for viewing the images. ${ }^{8}$ The increased contrast ratio and the presence of a unique front consistency sensor in MD also might have resulted in observer preference in favor of $\mathrm{MD}$

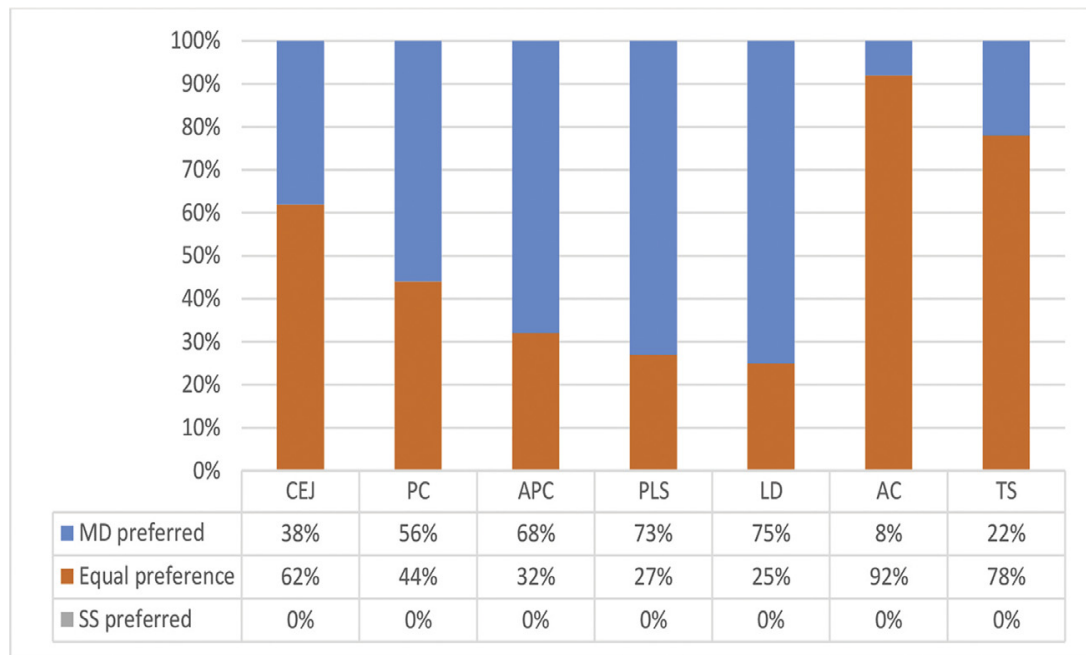

Fig. 8. In vivo radioanatomic feature detection on $\mathrm{CBCT}$ images. $A C$, alveolar crest; $A P C$, apical pulp canal; $C E J$, cementoenamel junction; $L D$, lamina dura; $M D$, medical display; $P C$, pulp canal; $P L S$, periodontal ligament space; $S S$, standard screen; $T S$, trabecular structure. 
compared with SS. SS had a higher pixel pitch and response time, but these parameters had no effect on the preference of the observers.

In previous studies, observers showed improved performance when utilizing MD for the detection of lung nodules and other complex medical anatomic structures. $^{12}$ However, most dental radiology studies showed better, but not significant, improvement in performance for measuring working length during endodontic treatment, detection of caries, and vertical root fractures when using display devices of varying quality. ${ }^{21-24}$ In contrast, our study showed significant increase in observer preference for MD because we focused on different radioanatomic features instead of pathologic lesions. Additionally, no studies were available in the literature comparing screen preferences for detecting multiple dental radioanatomic features. We believe our study fills the gap related to observers' preference of MD for detecting dental radioanatomic features.

The main limitation of our study was related to the number of screens included. Further studies should be conducted to compare the effectiveness of multiple dedicated MD and SS in detection of common pathologic conditions to observe whether MD with dentomaxillofacial settings can improve diagnostic efficacy. Also, the effects of medical monitor technology, such as in-plane switching and thin-film transistors, should also be investigated to assess which technology offers improved image preference with optimal quality in dentomaxillofacial radiology.

\section{CONCLUSIONS}

Overall, observers preferred the MD monitor over the standard monitor for both in vitro and in vivo observation of normal dental radioanatomic features irrespective of the imaging modality.

\section{REFERENCES}

1. Benson BW, Flint DJ, Liang H, Opatowsky MJ. Advances in diagnostic imaging for pathologic conditions of the jaws. Head Neck Pathol. 2014;8:383-391.

2. Suomalainen A, Pakbaznejad Esmaeili E, Robinson S. Dentomaxillofacial imaging with panoramic views and cone beam CT. Insights Imaging. 2015;6:1-16.

3. Longo DL, Fumes AC, De Oliveira DSB, et al. Comparison of digital and conventional radiographic techniques. RSBO. 2017;1:74-79.

4. Versteeg CH, Sanderink GCH, Van Der Stelt PF. Efficacy of digital intra-oral radiography in clinical dentistry. J Dent. 1997;25:215-224.

5. Gormez O, Yilmaz HH. Image post-processing in dental practice. Eur J Dent. 2009;3:343-347.

6. Choi JW, Han WJ, Kim EK. Image enhancement of digital periapical radiographs according to diagnostic tasks. Imaging $S c i$ Dent. 2014;44:31-35.

7. Nascimento MCC, Nejaim Y, De Almeida SM, et al. Influence of cone beam CT enhancement filters on diagnosis ability of longitudinal root fractures. Dentomaxillofac Radiol. 2014;43:20130374

8. Kagadis GC, Walz-Flannigan A, Krupinski EA, et al. Medical imaging displays and their use in image interpretation. RadioGraphics. 2013;33:275-290.

9. Samei E, Badano A, Chakraborty D, et al. Assessment of display performance for medical imaging systems: executive summary of AAPM TG18 report. Med Phys. 2005;32:1205-1225.

10. Indrajit I, Verma B. Monitor displays in radiology: Part 2. Indian J Radiol Imaging. 2009;19:94-98.

11. Lee KW, Wang AH, Fu JC, et al. Effect of the display medium of a mammogram on microcalcification-diagnostic performance and the subjective preference of radiologists according to differing professional experience. Displays. 2005;26:153-158.

12. Buls N, Shabana W, Verbeek P, Pevenage P, De Mey J. Influence of display quality on radiologists' performance in the detection of lung nodules on radiographs. Br J Radiol. 2007;80:738-743.

13. Butt A, Savage NW. Digital display monitor performance in general dental practice. Aust Dent J. 2015;60:240-246

14. Macdonald-Jankowski DS, Orpe EC. Some current legal issues that may affect oral and maxillofacial radiology. Part 2: Digital monitors and cone-beam computed tomography. J Can Dent Assoc. 73:507-511.

15. Mcllgorm D. Viewing your digital radiographs: which monitor is best? Br Dent J. 2016;220:393-397.

16. Jones DEA, Raine HC. A letter to the editor. Br J Radiol. 1949;22:549-550

17. McHugh ML. Interrater reliability: the kappa statistic. Biochem Medica. 2012;22:276-282.

18. Abdinian M, Soheilipour F, Nazeri R, Ghorbanizadeh S. Investigation of the magnification of digital panoramic radiographs in different regions of the jaws. SRM J Res Dent Sci. 2016;7:10.

19. Kallio-Pulkkinen S, Haapea M, Liukkonen E, Huumonen S, Tervonen O, Nieminen MT. Comparison of consumer grade, tablet and 6 MP-displays: observer performance in detection of anatomical and pathological structures in panoramic radiographs. Oral Surg Oral Med Oral Pathol Oral Radiol. 2014;118:135-141.

20. Patel KS, Kulkarni N, Singh VP, Parikh K. Identification of an alternate maxillary apical base landmark from pre-existing substitutions. Acta Inform Med. 2014;22:347-349.

21. Countryman SC, Sousa Melo SL, Belem MDF, Haiter-Neto F, Vargas MA, Allareddy V. Performance of 5 different displays in the detection of artificial incipient and recurrent caries-like lesions. Oral Surg Oral Med Oral Pathol Oral Radiol. 2018;125:182-191

22. Vasconcelos TV, Santaella GM, do Nascimento HAR, Rovaris K, Ambrosano GMB, Freitas DQ. Digital radiographs displayed on different devices: effect on the detection of vertical root fractures. Int Endod J. 2016;49:386-392.

23. Lally T, Geist JR, Yu Q, Himel VT, Sabey K. Evaluation of 4 commercial viewing devices for radiographic perceptibility and working length determination. J Endod. 2015;41:1120-1124.

24. Tofangchiha M, Adel M, Bakhshi M, et al. Digital radiography with computerized conventional monitors compared to medical monitors in vertical root fracture diagnosis. Iran Endod $\mathrm{J}$. 2013;8:14-17.

Reprint requests:

Sohaib Shujaat

OMFS-IMPATH Research Group

Campus Sint-Rafaël

Kapucijnenvoer 33

BE-3000 Leuven, Belgium

Sohaib.shujaat@kuleuven.be 